\title{
A COEFFICIENT PROBLEM OF BOMBIERI CONCERNING UNIVALENT FUNCTIONS
}

\author{
DAOUD BSHOUTY
}

\begin{abstract}
We answer affirmatively a question raised by Bombieri concerning the behaviour of the coefficients of normalized univalent functions near the Koebe function.
\end{abstract}

1. Introduction. Let $S$ denote the class of functions $f(z)=z+a_{2} z^{2}+\cdots$, regular and univalent in $\{z:|z|<1\}$. Bombieri $[\mathbf{1}]$ has shown that there exist constants $c_{n}$ such that

$$
\left|\operatorname{Re}\left(a_{n}-n\right)\right| \leq c_{n} \operatorname{Re}\left(2-a_{2}\right) .
$$

In $\left[2,\right.$. Problem 6.3] he posed the question whether there exist constants $d_{n}$ such that

$$
|| a_{n}|-n| \leq d_{n}\left(2-\left|a_{2}\right|\right) .
$$

This question is answered affirmatively and, in fact, we prove the following version:

$$
|| a_{n}|-n| \leq d_{n}\left(\operatorname{Re}\left(2-a_{2}\right)\right) \text {. }
$$

The equivalence of the last two inequalities is easily shown by noting that if $f(z)$ is in $S$, then the rotation $f_{\varphi}$ is in $S$, where

$$
f_{\varphi}(z)=e^{-i \varphi} f\left(e^{i \varphi} z\right)=z+e^{i \varphi} a_{2} z^{2}+e^{2 i \varphi} a_{3} z^{3}+\cdots .
$$

Inequalities (A) and (B) are equivalent to the following inequalities:

$$
\begin{gathered}
\operatorname{Re} a_{n}>n-c_{n}\left(\operatorname{Re}\left(2-a_{2}\right)\right), \\
\operatorname{Re} a_{n}<n+c_{n}\left(\operatorname{Re}\left(2-a_{2}\right)\right), \\
\left|a_{n}\right|>n-d_{n}\left(\operatorname{Re}\left(2-a_{2}\right)\right), \\
\left|a_{n}\right|<n+d_{n}\left(\operatorname{Re}\left(2-a_{2}\right)\right) .
\end{gathered}
$$

These inequalities are interrelated. $(\mathrm{Aa})$ implies $(\mathrm{Ba})$ and $(\mathrm{Bb})$ implies $(\mathrm{Ab})$. ( $\mathrm{Aa}$ ) describes how small $\operatorname{Re} a_{n}$ can be for functions close to the Koebe function, a result of which is of independent interest. $(\mathrm{Ab})$ is less significant since the stronger local Bierberbach conjecture $\operatorname{Re} a_{n}<n$ is known to be true for functions close to the Koebe function. As to (Bb), it is more significant, since the corresponding conjecture, namely $\left|a_{n}\right|<n$ for functions close to the Koebe function, is still unsolved. In fact, Bombieri himself considers this conjecture to be still open (private communication). However, in $[\mathbf{4}$, p. 83], there is an incorrect related statement, as the author claims that by a small rotation of the unit disk one may replace $\operatorname{Re} a_{n}$ by $\left|a_{n}\right|$ in the local Bierberbach conjecture. This is certainly false. Indeed, the strongest result of Bombieri stated in [5, p. 26] claims:

Received by the editors April 6, 1983.

1980 Mathematics Subject Classification. Primary 30C50.

(C) 1984 American Mathematical Society $0002-9939 / 84 \$ 1.00+\$ .25$ per page 
There are constants $\alpha_{n}$ and $\delta_{n}$ such that

$$
\operatorname{Re} a_{n}<n-\alpha_{n}\left(2-\operatorname{Re} a_{2}\right) \text { if } n \text { even, }\left|2-a_{2}\right|<\delta_{n} .
$$

If we apply this to a rotation we get

$$
\left|a_{n}\right|<n-\alpha_{n}\left|2-\operatorname{Re} a_{2} e^{i(\theta+2 j \pi) /(n-1)}\right| \quad \text { if } n \text { even, }\left|2-a_{2} e^{i(\theta+2 j \pi)(n-1)}\right|<\delta_{n},
$$

where $\theta=-\arg a_{n}, j$ natural. In particular,

$$
\left|a_{n}\right|<n-\alpha_{n}\left(2-\left|a_{2}\right|\right) \text { if } n \text { even, }\left|2-a_{2} e^{i(\theta+2 j \pi) /(n-1)}\right|<\delta_{n} .
$$

Noting that $\theta$ depends on the function itself, the class of univalent functions for which

$$
\left|2-a_{2} e^{i(\theta+2 \pi j) /(n-1)}\right|<\delta_{n}, \quad \text { for some natural } j,
$$

is only a subset of the class of univalent functions for which $|2-| a_{2}||<\delta_{n}$. This is why $(\mathrm{Bb})$ is not implied by $(\mathrm{C})$ applied to a rotation of the function.

In our proof of inequality (B), we will not find explicit bounds for $d_{n}$, although estimates could be obtained from the argument.

2. Main lemma. Our approach is a straightforward application of the Löwner differential equation theory. Let $T$ be the set of slit mappings $f(z)=z+a_{2} z^{2}+\cdots$, which can be imbedded in a Löwner chain $f(z, t)=e^{t} z+a_{2}(t) z^{2}+\cdots$ in the sense that

$$
f(z) \equiv f(z, 0)
$$

where $f(z, t)$ satisfies

$$
\frac{\partial f(z, t)}{\partial t}=z \frac{\partial f(z, t)}{\partial z} \cdot \frac{1+k(t) z}{1-k(t) z} \text { in }|z|<1, t>0,
$$

and $k(t)$ is a continuous function satisfying $|k(t)|=1$. In the topology of uniform convergence on closed subsets of $|z|<1, T$ is dense in $S$. Since $a_{j}$ is a continuous functional on $S$, it is enough to prove coefficient inequalities such as (A) and (B) for the class $T$. Often we will make use of the well-known recursion formulae for $a_{n}(t)$ (see, for example, $[\mathbf{5}$, Chapter 6]). We also note the special case $k(t) \equiv-1$ which gives rise to the chain $f(z, t)=e^{t} z /(1-z)^{2}=\sum_{n=1}^{\infty} n e^{t} z^{n}$.

Our aim is to estimate $a_{n}(t)$. We write

$$
k(s)=-e^{i \theta(s)}, \quad-\pi<\theta(s) \leq \pi, \quad \theta(s) \text { piecewise continuous. }
$$

Then

$$
\begin{aligned}
\operatorname{Re}\left(2 e^{t}-a_{2}(t)\right) & =2 e^{2 t} \int_{t}^{\infty}(1-\operatorname{Cos} \theta(s)) e^{-s} d s \\
& =4 e^{2 t} \int_{t}^{\infty} \operatorname{Sin}^{2} \frac{\theta(s)}{2} \cdot e^{-s} d s \leq e^{2 t} \int_{t}^{\infty} \theta^{2}(s) e^{-s} d s
\end{aligned}
$$

and

$$
\begin{aligned}
\left|\operatorname{Im} a_{2}(t)\right| & =\left|2 e^{2 t} \int_{t}^{\infty} \operatorname{Sin} \theta(s) \cdot e^{-s} d s\right| \\
& \leq 2 e^{2 t} \int_{t}^{\infty}|\theta(s)| e^{-s / 2} \cdot e^{-s / 2} d s \leq 2 e^{3 t / 2}\left(\int_{t}^{\infty} \theta^{2}(s) e^{-s} d s\right)^{1 / 2}
\end{aligned}
$$

by the Schwarz inequality. 
We shall deduce

LEMMA. Let $n \geq 2$. There exist constants $\alpha_{n}$ and $\beta_{n}$ such that

$$
\begin{aligned}
& \left|\operatorname{Re} a_{n}(t)-n e^{t}\right| \leq \alpha_{n} e^{2 t} \int_{t}^{\infty} \theta^{2}(s) e^{-s} d s, \\
& \left|\operatorname{Im} a_{n}(t)\right| \leq \beta_{n} e^{3 t / 2}\left(\int_{t}^{\infty} \theta^{2}(s) e^{-s} d s\right)^{1 / 2} .
\end{aligned}
$$

PROOF. This is certainly true for $n=2$ with $\alpha_{2}=1$ and $\beta_{2}=2$ by (3) and (4). We proceed by induction. We assume these inequalities are true for $n=2,3, \ldots, m-1$. Now

$$
a_{m}(t)=-2 e^{m t} \sum_{\nu=1}^{m-1} \nu \int_{t}^{\infty} e^{-m s} k_{1}(s)^{m-\nu} \cdot a_{\nu}(s) d s
$$

and

$$
m e^{t}=-2 e^{m t} \sum_{\nu=1}^{m-1} \nu \int_{t}^{\infty} e^{-m s}(-1)^{m-\nu} \cdot \nu e^{s} d s .
$$

To estimate the required quantities we first note the following:

$k(s)^{m-\nu} a_{\nu}(s)-(-1)^{m-\nu} \nu e^{s}=\left(k(s)^{m-\nu}-(-1)^{m-\nu}\right) a_{\nu}(s)+(-1)^{m-\nu}\left(a_{\nu}(s)-\nu e^{s}\right)$,

so that

$$
\begin{aligned}
\operatorname{Re}\left(k(s)^{m-\nu} a_{\nu}(s)-(-1)^{m-\nu} \nu e^{s}\right) & \\
=(-1)^{m-\nu} \cdot\left(\left(\operatorname{Re} e^{i(m-\nu) \theta(s)}-1\right) \cdot \operatorname{Re} a_{\nu}(s)\right. & \\
& \left.-\operatorname{Im} e^{i(m-\nu) \theta(s)} \cdot \operatorname{Im} a_{\nu}(s)+\left(\operatorname{Re} a_{\nu}(s)-\nu e^{s}\right)\right)
\end{aligned}
$$

and thus, using (3), (4) and the induction assumption, we have

$$
\begin{aligned}
\left|\operatorname{Re}\left(k(s)^{m-\nu} a_{\nu}(s)-(-1)^{m-\nu} \nu e^{s}\right)\right| & \\
\leq & \frac{(m-\nu)^{2}}{4} \cdot \theta^{2}(s) \cdot\left(\nu e^{s}+\alpha_{\nu} e^{2 s} \int_{s}^{\infty} \theta^{2}(\sigma) e^{-\sigma} d \sigma\right) \\
& +(m-\nu)|\theta(s)| \beta_{\nu} e^{3 s / 2}\left(\int_{s}^{\infty} \theta^{2}(\sigma) e^{-\sigma} d \sigma\right)^{1 / 2}+\alpha_{\nu} e^{2 s} \int_{s}^{\infty} \theta^{2}(\sigma) e^{-\sigma} d \sigma \\
\leq & m^{3} \cdot e^{2 s}\left\{\left[\left(1+\theta^{2}(s)\right) \alpha_{\nu} \int_{s}^{\infty} \theta^{2}(\sigma) e^{-\sigma} d \sigma+\theta^{2}(s) e^{-s}\right]\right. \\
& \left.+|\theta(s)| \beta_{\nu} e^{-s / 2}\left(\int_{s}^{\infty} \theta^{2}(\sigma) e^{-\sigma} d \sigma\right)^{1 / 2}\right\} .
\end{aligned}
$$

Also note that

$$
\operatorname{Im}\left(k(s)^{m-\nu} a_{\nu}(s)\right)=\operatorname{Re} k(s)^{m-\nu} \cdot \operatorname{Im} a_{\nu}(s)+\operatorname{Im} k(s)^{m-\nu} \cdot \operatorname{Re} a_{\nu}(s) .
$$


Thus, using the same arguments, we deduce

$$
\begin{aligned}
& \left|\operatorname{Im}\left(k(s)^{m-\nu} \cdot a_{\nu}(s)\right)\right| \\
& \leq\left(1+m^{2} \cdot \theta^{2}(s)\right) \beta_{\nu} e^{3 s / 2}\left(\int_{s}^{\infty} \theta^{2}(\sigma) e^{-\sigma} d \sigma\right)^{1 / 2} \\
& \quad+m|\theta(s)|\left(\nu e^{s}+\alpha_{\nu} e^{2 s} \int_{s}^{\infty} \theta^{2}(\sigma) e^{-\sigma} d \sigma\right) \\
& \leq m^{2} \cdot e^{3 s / 2}\left\{\left(1+\theta^{2}(s)\right) \beta_{\nu}\left(\int_{s}^{\infty} \theta^{2}(\sigma) e^{-\sigma} d \sigma\right)^{1 / 2}\right. \\
& \left.\quad+|\theta(s)| e^{-s / 2}\left(\nu+\alpha_{\nu} e^{s} \int_{\sigma}^{\infty} \theta^{2}(\sigma) e^{-\sigma} d \sigma\right)\right\} .
\end{aligned}
$$

We conclude that

$$
\begin{aligned}
\left|\operatorname{Re} a_{m}(t)-m e^{t}\right| & \leq 2 m^{3} e^{m t} \sum_{\nu=1}^{m-1} \nu \int_{t}^{\infty} e^{(-m+2) s} \\
\cdot\{ & \left\{\left(1+\theta^{2}(s)\right) \alpha_{\nu} \int_{s}^{\infty} \theta^{2}(\sigma) e^{-\alpha} d \sigma+\theta^{2}(s) e^{-s}\right] \\
& \left.+|\theta(s)| e^{-s / 2} \beta_{\nu}\left(\int_{s}^{\infty} \theta^{2}(\sigma) e^{-\sigma} d \sigma\right)^{1 / 2}\right\} d s
\end{aligned}
$$

and

$$
\begin{aligned}
&\left|\operatorname{Im} a_{m}(t)\right| \leq 2 m^{2} e^{m t} \sum_{\nu=1}^{m-1} \nu \int_{t}^{\infty} e^{(-m+3 / 2) s} \\
& \cdot\left[\left(1+\theta^{2}(s)\right) \beta_{\nu}\left(\int_{s}^{\infty} \theta^{2}(\sigma) e^{-\sigma} d \sigma\right)^{1 / 2}\right. \\
&\left.\quad+|\theta(s)| e^{-s / 2}\left(\nu+\alpha_{\nu} e^{s} \int_{s}^{\infty} \theta^{2}(\sigma) e^{-\sigma} d \sigma\right)\right] d s .
\end{aligned}
$$

The integrals involved in the first inequality are of the form

$$
\int_{t}^{\infty} e^{(-m+2) s} \gamma_{\nu}(\theta)\left(\int_{s}^{\infty} \theta^{2}(\sigma) e^{-\sigma} d \sigma\right) d s, \quad \int_{t}^{\infty} e^{(-m+2) s} \theta^{2}(s) e^{-s} d s
$$

and

$$
\int_{t}^{\infty} e^{(-m+2) s}|\theta(s)| e^{-s / 2}\left(\int_{s}^{\infty} \theta^{2}(\sigma) e^{-\sigma} d \sigma\right)^{1 / 2} d s
$$

where $\gamma_{\nu}(\theta)=\left(1+\theta^{2}(s)\right) \alpha_{\nu}$ for $1 \leq \nu \leq m-1$, all of which are bounded by

$$
c_{\nu} e^{(-m+2) t} \int_{t}^{\infty} \theta^{2}(s) e^{-s} d s
$$


(here and henceforth, the symbol $c_{\nu}$ refers to indefinite constants depending on $\nu$ ). This is easily verified. In the first case note that $\gamma_{\nu}(\theta) \leq c_{\nu}$ and $m \geq 3$, so

$$
\begin{aligned}
\int_{t}^{\infty} & e^{(-m+2) s} \gamma_{\nu}\left(\int_{s}^{\infty} \theta^{2}(\sigma) e^{-\sigma} d \sigma\right) d s \\
& \leq c_{\nu} \int_{t}^{\infty} e^{(-m+2) s}\left(\int_{t}^{\infty} \theta^{2}(\sigma) e^{-\sigma} d \sigma\right) d s \\
& \leq c_{\nu} e^{(-m+2) t} \int_{t}^{\infty} \theta^{2}(\sigma) e^{-\sigma} d \sigma
\end{aligned}
$$

In the second case note that $e^{(-m+2) s} \leq e^{(-m+2) t}$ for $s \geq t \geq 0$, whereas in the third case

$$
\begin{aligned}
\int_{t}^{\infty} & e^{(-m+2) s}|\theta(s)| e^{-s / 2}\left(\int_{s}^{\infty} \theta^{2}(\sigma) e^{-\sigma} d \sigma\right)^{1 / 2} d s \\
& \leq\left(\int_{t}^{\infty} \theta^{2}(\sigma) e^{-\sigma} d \sigma\right)^{1 / 2} \int_{t}^{\infty} e^{(-m+2) s}|\theta(s)| e^{-s / 2} d s \\
& \leq e^{(-m+2) t} \int_{t}^{\infty} \theta^{2}(\sigma) e^{-\sigma} d \sigma
\end{aligned}
$$

Note in the last step the Schwarz inequality has been applied to the second factor.

The integrals involved in the second inequality are of the form

$$
\int_{t}^{\infty} e^{(-m+3 / 2) s} \delta_{\nu}(\theta)\left(\int_{s}^{\infty} \theta^{2}(\sigma) e^{-\sigma} d \sigma\right)^{1 / 2} d s
$$

and

$$
\int_{t}^{\infty} e^{(-m+3 / 2) s}|\theta(s)| e^{-s / 2} \eta_{\nu}(s) d s
$$

where

$$
\delta_{\nu}(\theta)=\left(1+\theta^{2}(s)\right) \beta_{\nu} \leq c_{\nu}
$$

and

$$
\eta_{\nu}(s)=\beta_{2}\left(\nu+\alpha_{\nu} e^{s} \int_{s}^{\infty} \theta^{2}(\sigma) e^{-\sigma} d \sigma\right)^{1 / 2} \leq c_{\nu}
$$

since $\theta^{2}(s)=O(\varepsilon)$. Both integrals are bounded by

$$
c_{\nu} e^{(-m+3 / 2) t}\left(\int_{t}^{\infty} \theta^{2}(s) \theta^{-s} d s\right)^{1 / 2} .
$$

To check this for the first integral, just increase its value by integrating inside from $t$ instead of from $s$. In the second integral use the Schwarz inequality.

Substituting these estimates in the corresponding inequalities, we obtain the conclusion of the Lemma.

3. Conclusion. We are ready now for our final result:

THEOREM. There exist constants $b_{n}, c_{n}, d_{n}$ such that for $n \geq 2$,

$$
\begin{aligned}
\left|a_{n}-n\right| & \leq b_{n}\left(\operatorname{Re}\left(2-a_{2}\right)\right)^{1 / 2}, \\
\left|\operatorname{Re} a_{n}-n\right| & \leq c_{n}\left(\operatorname{Re}\left(2-a_{2}\right)\right),
\end{aligned}
$$


(8)

$$
|| a_{n}|-n| \leq d_{n}\left(\operatorname{Re}\left(2-a_{2}\right)\right) .
$$

Proof. From (1), we have $a_{n}=a_{n}(0)$, so

$$
\begin{aligned}
\operatorname{Re}\left(2-a_{2}\right) & =2 \int_{0}^{\infty}(1-\operatorname{Cos} \theta(s)) e^{-s} d s \\
& =4 \int_{0}^{\infty} \operatorname{Sin}^{2} \frac{\theta(s)}{2} \cdot e^{-s} d s \geq \frac{4}{\pi^{2}} \int_{0}^{\infty} \theta^{2}(s) e^{-s} d s
\end{aligned}
$$

Also,

$$
\begin{aligned}
\left|a_{n}-n\right| & \leq\left|\operatorname{Re} a_{n}-n\right|+\left|\operatorname{Im} a_{n}\right| \\
& \leq \alpha_{n} \int_{0}^{\infty} \theta^{2}(s) e^{-s} d s+\beta_{n}\left(\int_{0}^{\infty} \theta^{2}(s) e^{-s} d s\right)^{1 / 2} \text { by }(5), \\
& \leq\left(\alpha_{n} \pi+\beta_{n}\right) \cdot\left(\int_{0}^{\infty} \theta^{2}(s) e^{-s} d s\right)^{1 / 2} \text { since } \int_{0}^{\infty} \theta^{2}(s) e^{-s} d s \leq \pi^{2},
\end{aligned}
$$

which, in conjunction with (9), proves (6). Inequality (7) is now immediate from (5). Inequality (8) is a consequence of the following inequality.

$$
\begin{aligned}
|| a_{n}|-n| & =\frac{1}{\left|a_{n}\right|+n}\left\{\left(\operatorname{Re} a_{n}+n\right)\left(\operatorname{Re} a_{n}-n\right)+\left(\operatorname{Im} a_{n}\right)^{2}\right\} \\
& \leq\left|\operatorname{Re} a_{n}-n\right|+\left(\operatorname{Im} a_{n}\right)^{2} .
\end{aligned}
$$

Then (6) and (7) imply (8) and the Theorem is proved.

REMARK 1 . It can be shown that the power $1 / 2$ in the Theorem cannot be relaxed. For starlike functions the exact power is 1 . In this case the exact value for $b_{n}$ is known $[3]$.

\section{REFERENCES}

1. E. Bombieri, Sull'integrazione approssimata dell' equazione differentiale di Löwner e le sue applicazioni alla teoria delle funzioni univalenti, Thesis, Milano, 1963.

2. W. K. Hayman, Research problems in function theory, Athlone Press, Belfast, 1967.

3. J. A. Hummel, The coefficients of starlike functions, Proc. Amer. Math. Soc. 22 (1969), 311-315.

4. I. M. Milin, Univalent functions and orthonormal systems, Transl. Math. Mono., Vol. 49, Amer. Math. Soc., Providence, R.I., 1977.

5. Chr. Pommerenke, Univalent functions, Vandenhoeck \& Ruprecht, Göttingen, 1975.

DEPARTMENT OF MATHEMATICS, TECHNION-ISRAEL INSTitute OF TEChNOLOGy, HAIFA, ISRAEL 